\title{
Research Paper \\ Psychometric Properties of the Persian Version of the Family Empowerment Scale
}

\author{
${ }^{*}$ Gholamreza Rajabi ${ }^{1} \odot$, Zahra Zolmajd ${ }^{2}$
}

1. PhD in Psychology, Professor, Department of Counseling, Faculty of Education and Psychology, Shahid Chamran University of Ahvaz, Ahvaz, Iran. 2. MSc., Department of Counseling, Faculty of Education and Psychology, Shahid Chamran University of Ahvaz, Ahvaz, Iran.

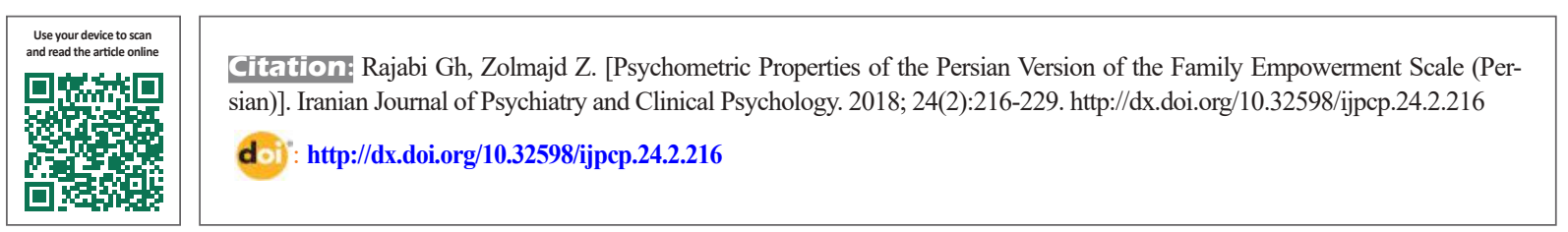

Received: 18 Oct 2017 Accepted: 14 Mar 2018

Key words: Psychometric, Empowerment, Family, Hyperactivity/attention deficit, Autism spectrum, Specific Learning

\section{A B STRACT}

Objectives The aim of the current research was to investigate the psychometric properties of the Persian version of the Family Empowerment Scale in families with children suffering from hyperactivity/attention deficit, autism spectrum and specific learning disorders.

Methods A total of 210 parents of exceptional children with the training settings from educational region 1 and 2 in Ahvaz city were selected using based-purpose and voluntary sampling method and responded to the Family Empowerment and Marital Satisfaction scales. The data were analyzed using exploratory factor analysis and statistical software SPSS- 21.

Results The findings revealed that the scale consisted of three factors - empowerment with respect to family system, service system and social/politics system. The Cronbach's alpha coefficients obtained 0.94 for the whole scale and ranged from 0.91 to 0.93 for the three factors, test-retest reliability coefficient was 0.86 and showed the convergent validity coefficient of $0.31(P<0.001)$ between the current scale and the Marital Satisfaction Scale.

Conclusion According to the results from this scale it can be used as an appropriate tool for measuring the ability of the parents to give services to their children with hyperactivity/attention deficit, autism spectrum and specific learning in exceptional rehabilitation settings and for identifying and explaining antecedents and consequences of this type of disorders.

\section{Extended Abstract}

\section{Introduction}

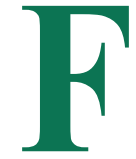

amily empowerment is increasingly seen as a central goal of efforts to improve services for the families where children have disabilities [1]. Empowerment is a general principle for promoting health and is relevant in all the areas of the community [2] and focuses on empowerment of family's abilities rather than deficiencies
[5]. The family empowerment concept has also took advantages from the synergistic effect of various disciplines focusing on one idea at the same time. Empowerment has been described as both a process and a state, both an individual and collective characteristic, as well as an attitude, perception, ability, knowledge and action, and finally as a phenomenon that can be manifested in a range of circumstances and environments. Family empowering is the potential to alter the family structure. Gutierrez and Ortega introduced three measures to assess different aspects of empowerment among Latinos. Two focused on political em-

* Corresponding Author:

Gholamreza Rajabi, PhD

Address: Department of Counseling, Faculty of Education and Psychology, Shahid Chamran University of Ahvaz, Ahvaz, Iran.

Tel: +98 (16) 33331366

E-mail: rajabireza@scu.ac.ir 
powerment, commitment and ethnic activism and the third on personal empowerment, conceptualized as a change strategy [7]. Staples asserted that, "In addition to transformations in consciousness, beliefs and attitudes, empowerment requires practical knowledge, solid information, real competencies, concrete skills, material resources, genuine opportunities and tangible results" [8].

The framework consisted of two dimensions: 1) The level of empowerment; and 2) The way that empowerment is expressed. Regarding the first dimension, empowerment can happen at three levels: 1) Family, i.e. the immediate situation at home that primarily involves the parent's management of day-to-day situations; 2) Service system, i.e., professionals and agencies that provide services to the parent's children that primarily involves the parent's active working with the service system to get needed services for their child; and 3) Community/political, i.e., legislative bodies, policy makers, agencies, and community members concerned with or influencing the services for children with emotional disorders and their families [8].

Regarding the second dimension, empowerment can be expressed in three ways: 1) Attitudes, i.e., what a parent feels and believes; 2) Knowledge, i.e., what a parent knows and can potentially do; and 3) Behavior, i.e., what a parent actually does. Each expression can occur within each dimension level. For example, empowerment with respect to the service system can be exhibited as an attitude, e.g. "Professionals should ask me about services I want for my child"; as knowledge, e.g. "I know what measures to be taken when I am concerned about receiving my child's poor services"; and as a behavior, e.g. "I inform professionals about my opinion on services being provided to my child." Thus, combining three categories with two dimensions results in nine possible combinations, displaying empowerment being experienced or expressed at each time [8].

Koren, DeChillo, and Friesen [8] first developed the Family Empowerment Scale. Few studies have been conducted to assess and measure the reliability and validity of this scale. Studies conducted by Singh, et al., [9]; Florian and Elad [10]; Pauline, et al. [11], Vuorenma, et al. [2], Wakimizu, et al. [12], Khalati, Nefei, and Soltani [13], and Kageyama, et al. [14] all indicated the reliability and validity of this scale. However, the aim of the current research was to investigate psychometric properties of the Persian version of the Family Empowerment Scale in families with children having hyperactivity, autism, and learning disorders.

\section{Method}

The present research is a psychometric and methodological study. A total of 210 parents among 470 families with children having hyperactivity/attention deficit, autism spectrum and specific learning disorders based on the inclusion criteria were taken for this study. The inclusion criteria are: the length of the marriage of parents between 1 and 15 years, the tendency to participate in the study, having a minimum age of 25 to 45 years, having no marital problems, not having mood and affective problems and not using the substances like drugs were selected by base-aimed and voluntary sampling method from the children's centers at the $1^{\text {st }}$ and $2^{\text {nd }}$ areas of education (Shakerin, Ehsan, Bahar Living, Tolo, Misagh, Nahal, Golshan, and Kosar) from January to May 2015-2016. To measure the family empowerment of Family Empowerment Scale the Korn and colleagues in 1992 [1] and to assess the convergent validity of this scale of the Marital Satisfaction Scale, the Rajabi was used [15].

\section{Results}

Most of the participants in this study were female parents $(54.8 \%)$, having a diploma degree $(32.9 \%)$ and had 2 children $(41.4 \%)$. The mean and standard deviation of 30 items of the Family Empowerment Scale were 105.85 and 22.33. For factor analysis the Family Empowerment Scale was used as the first principle component analysis and then to determine the underlying factors for the test of Varimax rotation that is an orthogonal rotation method. The 3-factor model with factor loadings higher of 0.40 (based on the exact cutoff point of the 3 factors of the Screen test) gave the analysis that the percentage of variance explained the Family Empowerment Scale items by the removal of 4 items $(3,13$, $26,29) 53.15$ was obtained. Finally, by removing these four items, a factor analysis was performed on 30 items of Family Empowerment Scale and the results obtained were KaiserMayer-Olkin sampling test $(\mathrm{KMO}=0.94)$ and Bartlett's Test of Sphericity $\left(\chi^{2}=3807.24\right)$, which explained 3-factor model $60 \%$ of variance of the scale items. In this factor analysis, the first factor of "empowerment with respect to the family system" has about $38.19 \%$; the most share in explaining the variance of the above scale (items 2, 4, 7, 9, 16, 21, 27, 34 31, $33)$. The second factor was labeled "empowerment with respect to the service system" with a loading of factor $13.08 \%$ (items 1, 5, 6, 11, 12, 18, 19, 23, 28, 30, 32). The third factor, empowerment with respect to the social/political system, was obtained with a variance of $8.72 \%$.

The results of Cronbach's alpha coefficients for the whole scale were 0.94 and for the three factors extracted from the exploratory factor structure ranged from 0.91 to 0.93 ; the test-retest reliability coefficient of this scale on 92 subject 
(with a 2-week interval) was $0.86(\mathrm{P}<0.001)$; convergent validity coefficients between Family Empowerment Scale and Marital Satisfaction Scale was 0.31, The convergent validity coefficients among three factors of family empowerment scale (empowerment respect to family system, empowerment respect to system of services and empowerment with respect to social/political system) and marital satisfaction scale was from 0.22 to $0.27(\mathrm{P}<0.001)$.

\section{Discussion}

The aim of this study was to investigate the psychometric properties of the Persian version of the Family Empowerment Scale in families with children suffering from hyperactivity, autism and learning disorders. The exploratory factor analysis of this scale showed three factors of empowerment with regard to the family system: empowerment with regard to service and empowerment system with regards to the social/political system, that this results in studies in consistence with Koren, et al. [1], Vuorenma, et al., [2] and Wakimizu, et al. [12]

The first factor with 10 items with the title empowerment in the family indicates that parents are equipped with childrearing practices and the ability to manage problems in everyday situations, which has led parents to adopt new ways to grow their children. They feel good about parenthood with their parents' knowledge and awareness of their ability to solve problems etc. The second factor was with 11 items of empowerment related to the service system, with parents' knowledge and information in working with services for children with developmental disorders and specialists to provide services for their children, the parents' attitude to the need for their child's services approval and their opinions are as important as experts; communication with professionals to collaborate on their relation to child's services for more information on child disorder and parents' knowledge when dealing with child problems, decisions to problems, sharing your view with experts and getting ahead included to have their child's services better [1].

The third factor was with 9 items empowerment in relation to the social/political system's ability, which to take parental actions to defend or improve services for children and families, their attitude towards their effectiveness on services that felt helpful in relation to their child's problem, knowledge about their rights and their children in the educational system, encouraging executives or legislators to listen to their conversations [1]. Evidence showed that problem some of the families are worried about obtaining appropriate services for their children. It is necessary to provide solid information that the increase in one level of empowerment stimulates other levels, or in other words whether empowering at one level would increase it at other levels or be separated from each other.

\section{Ethical Considerations}

\section{Compliance with ethical guideline}

Parents of Autism Children were selected with complete consent, knowing what this research was and what they should answer.

\section{Funding}

This article was extracted from a MA thesis in Family Counseling with the permission of Shahid Chamran University's Research Deputy of Ahvaz.

\section{Conflict of interest}

The authors declared no conflict of interest.

\section{Acknowledgments}

We sincerely thank the cooperation of the management of the exceptional children's educational centers of Ahvaz and all the parents who provided the opportunity to do this research. 


\title{
ويثَّى هاى روانسنجى نسخه فارسى مقياس توانمندسازى خانواده
}

\author{
•غلامرضا رجبى'، زهرا ذوالمجد‘
}

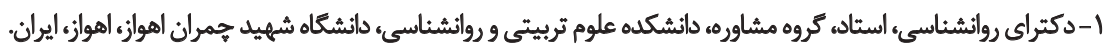

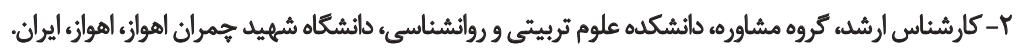

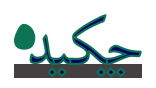

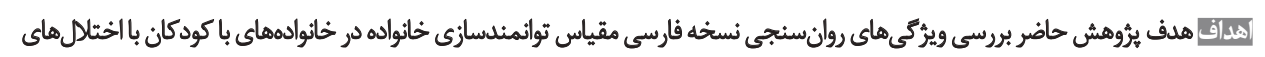

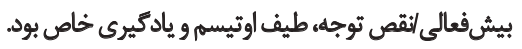

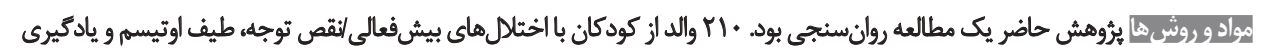

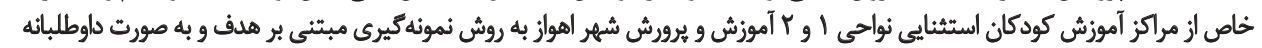

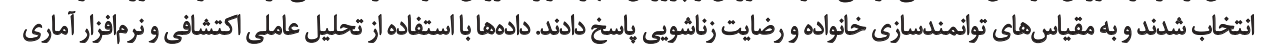

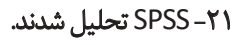

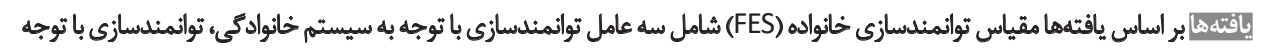

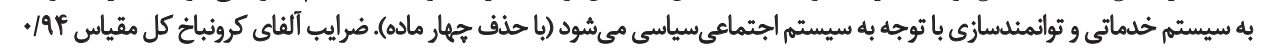

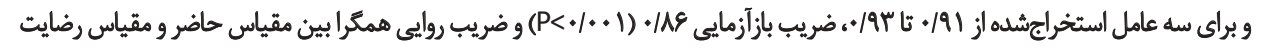

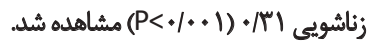

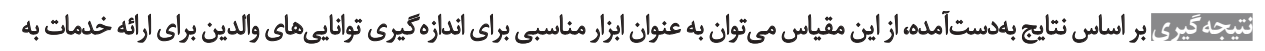

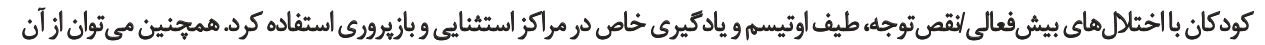

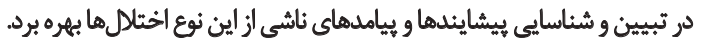

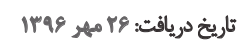

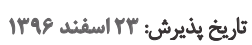

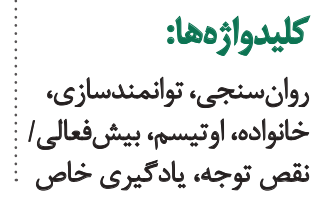

كليدوازٔها:

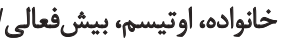
نقص توجه، يادكيرى خاص باصل

توانمندسازى خانواده، ايجاد توانايى تغيير در ساختار خانواده

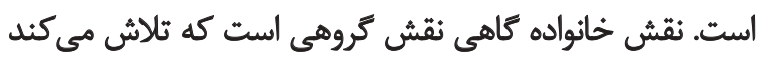

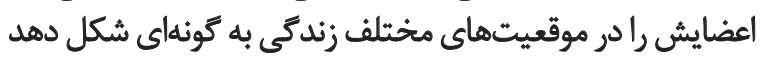

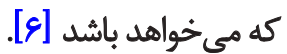

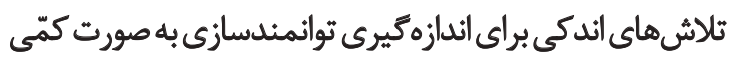

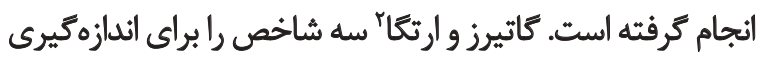

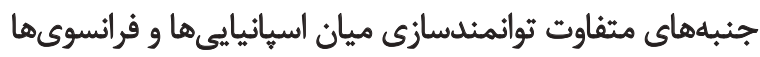

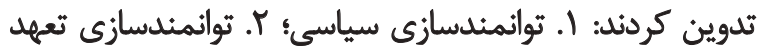

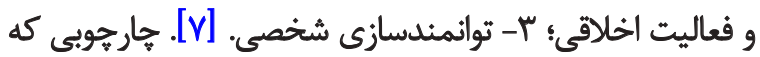

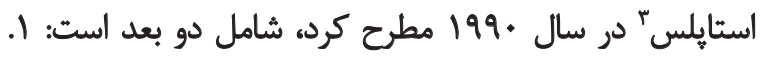

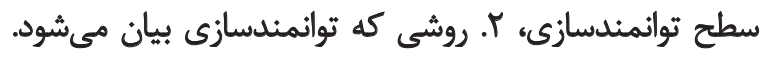

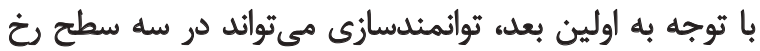

2. Gutierrez \& Ortega

توانمندسازى خانواده' به طور فزايندهاى هدف اصلى در

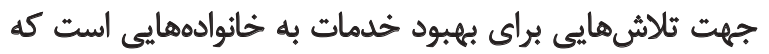

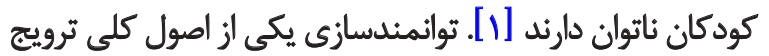

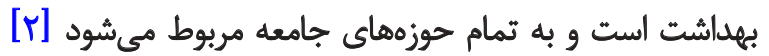

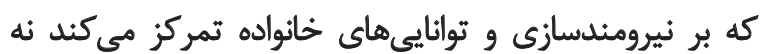

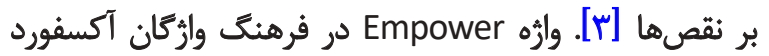

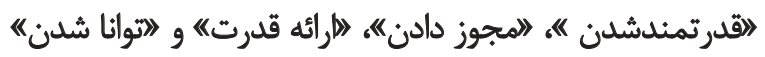

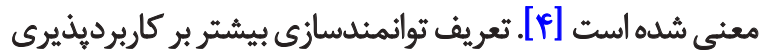

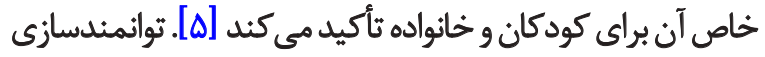

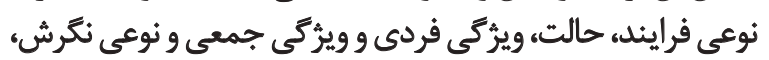

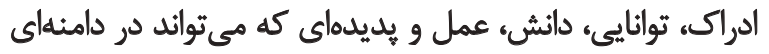

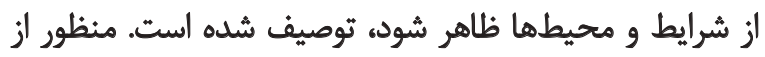

1. Family empowerment

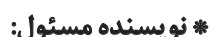

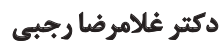

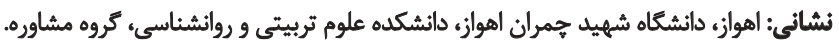
تلفن: rajabireza@scu.ac.ir يست الكترونيكي 


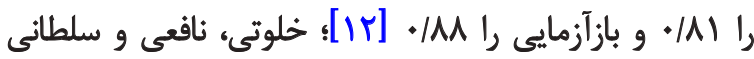

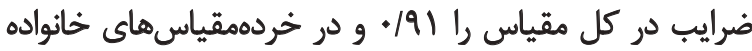

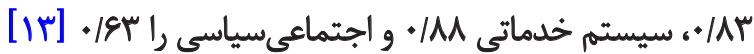

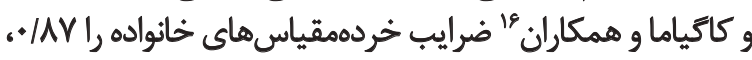

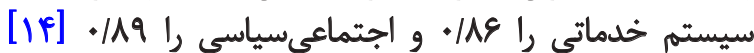

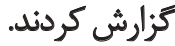

كورن و همكاران در مطالعهاى از نوع تحليل عاملى اكتشافى ترانى

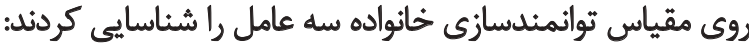

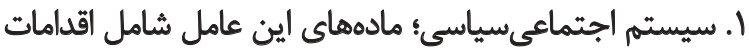

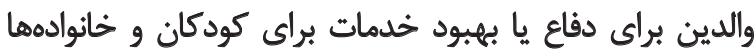

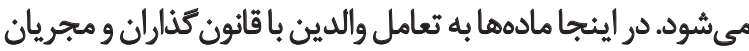

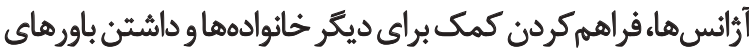

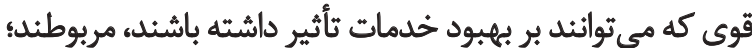

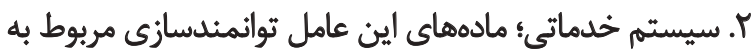

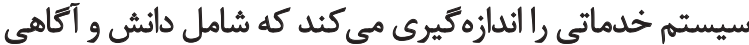

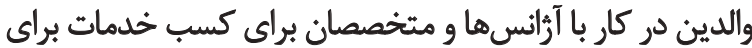

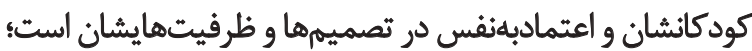

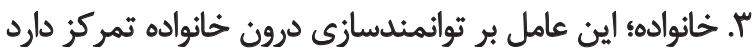

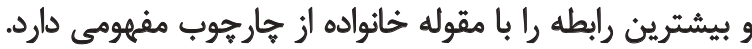

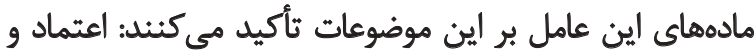

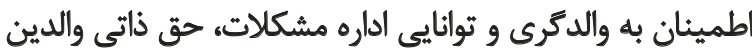

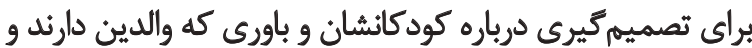

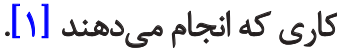

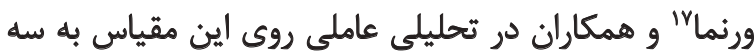

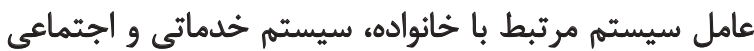

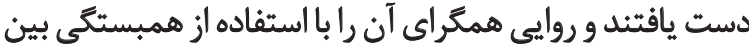

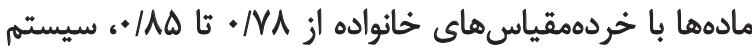

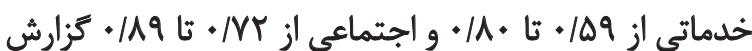

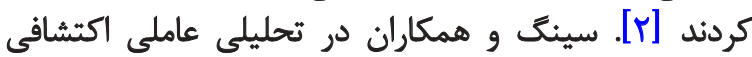

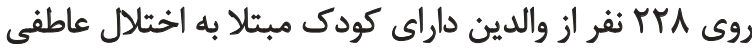

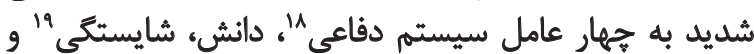

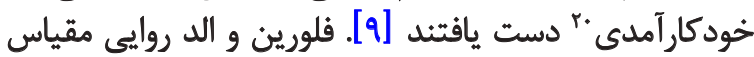

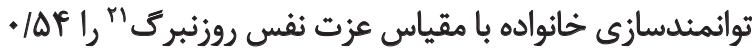

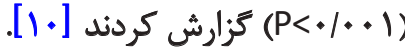

خائوادههايى كه يك يا جند عضو معلول دارند و خانوادههاي

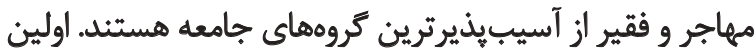

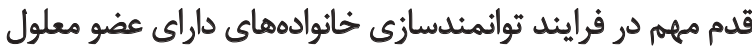

16. Nakamura, Kobayashi, \& Yokoyama

17.Vuorenma

18. Advocacy system

19. Competence

20. Self-efficacy

21. Rosenberg Self-Esteem Scale
دهد: الف) خانواده"، يعنى وضعيت فورى و بحرانى در خانه. اين اين

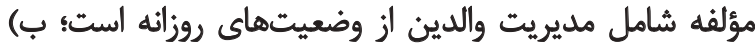

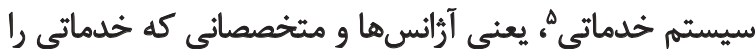

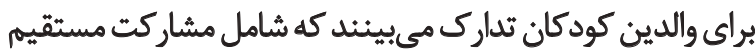

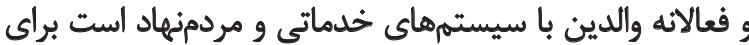
دستيابى به خدماتيى كه كودى او نيازمند است؛ جاني ج) سيسته

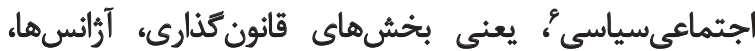

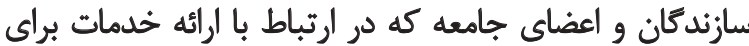

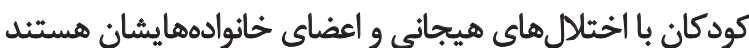

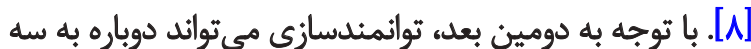

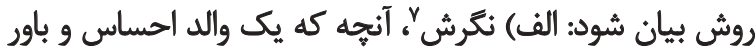

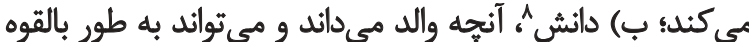

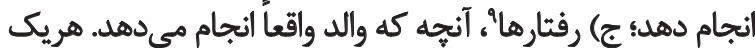

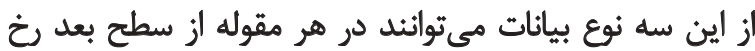

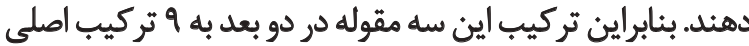

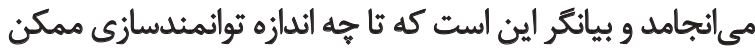
است در مقطع زمانى معينى تجربه يا بيان شود [N]]

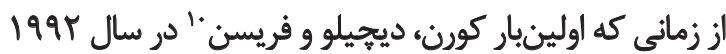

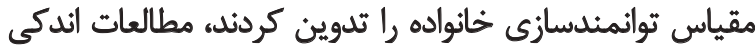

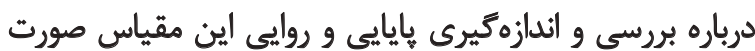

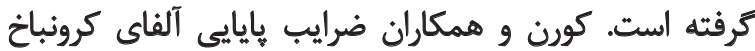

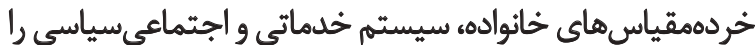

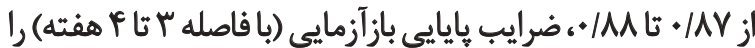

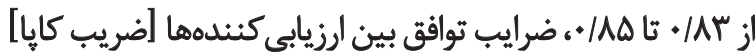

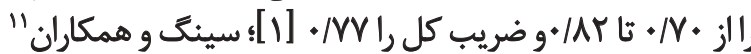

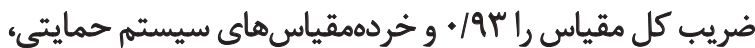

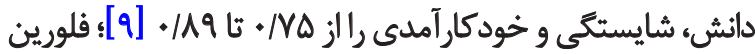

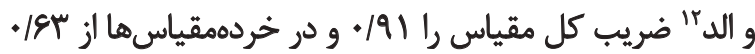

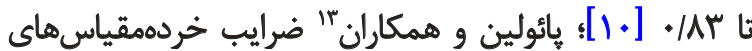

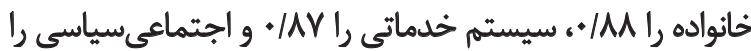

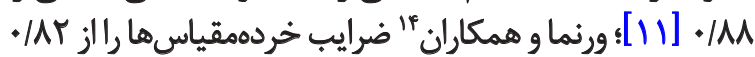

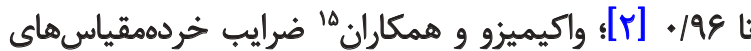

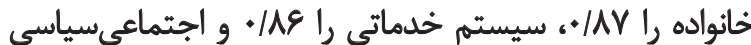

4. Family

5. Service

6. Community/political

7. Attitude

8. Knowledge

9. Behavior

10. Koren, DeChillo \& Friesen

11. Singh

12. Florian \& Elad

13. Pauline

14. Vuorenma et al.

15. Yoneyama, lejima, \& Miyamoto 
ملاقات ضرورت و اهميث اين برؤهش و تمامى مسائل مربوط

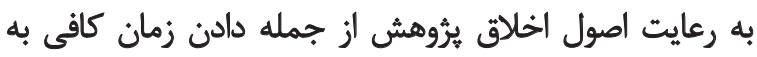

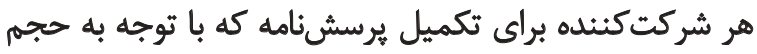

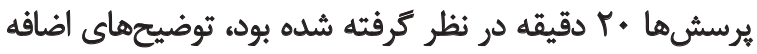

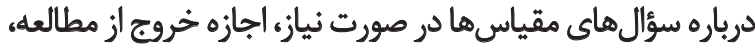

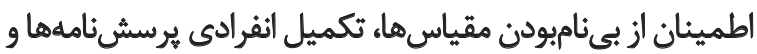

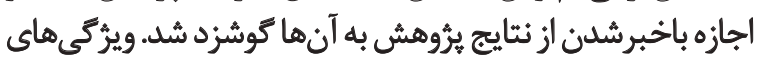

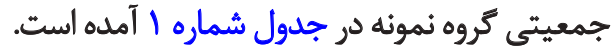

براى اندازهيرى توانمندسازى خانواده از مقياس توانمندسازى خانواده كورن و همكاران استفاده شد كه در سال

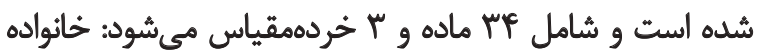

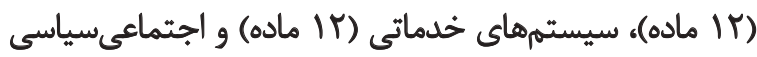

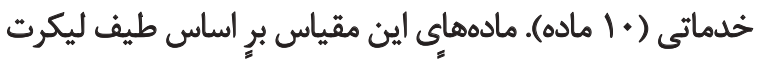

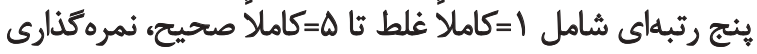

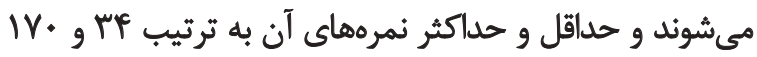

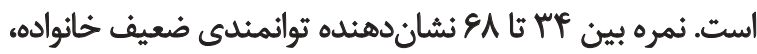

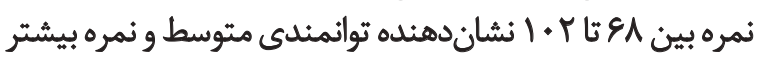

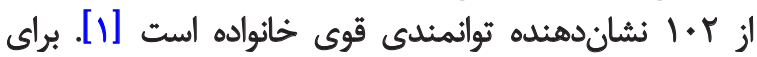

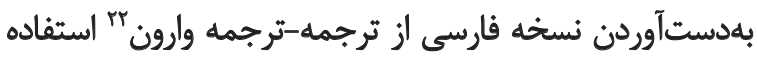

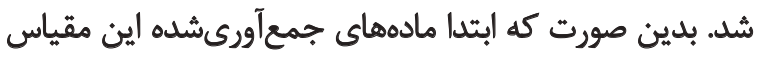

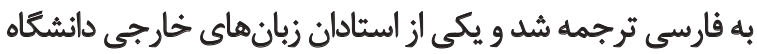

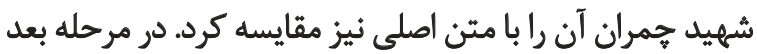

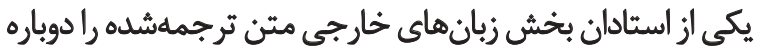

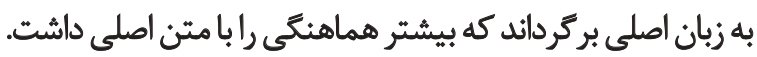

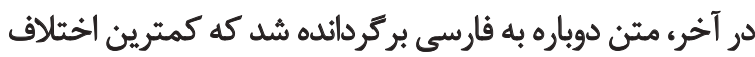

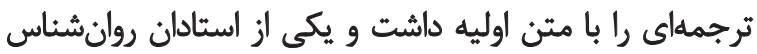
دانشكاهي از نظر محتواي مادهها آن را الرزيابي كرد.

براى اندازهكيرى روايى همكرا مقياس توانمندسازى خانواده

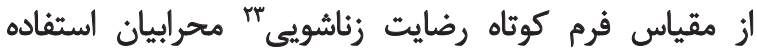

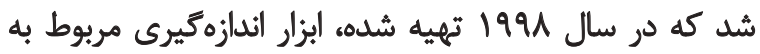

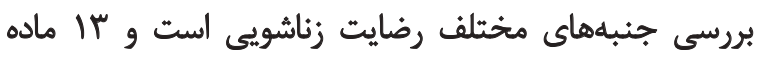

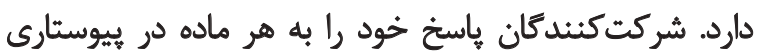

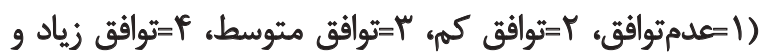

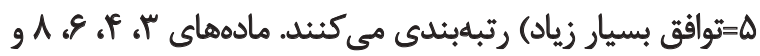

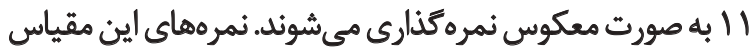

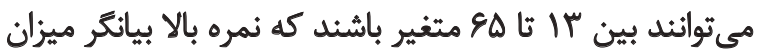

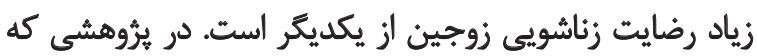

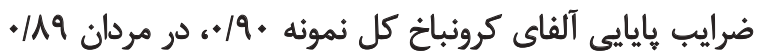

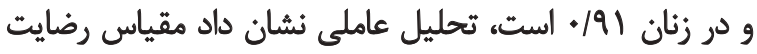

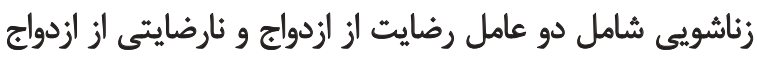

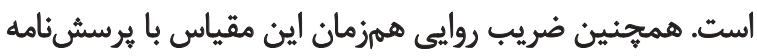

22. Back translation

23. Marital Satisfaction Scale (MSS)
اين است كه متخصص تشخيص دهد والدين، شايستئى و درى

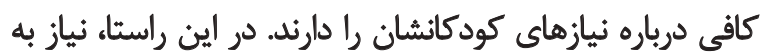

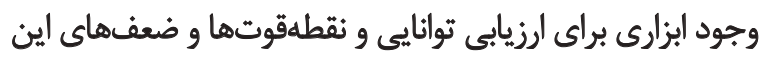

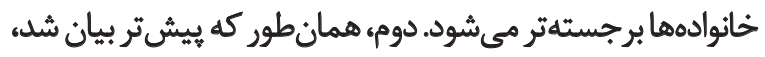

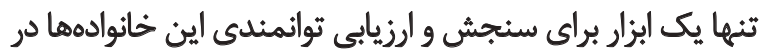

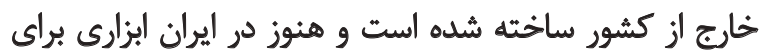

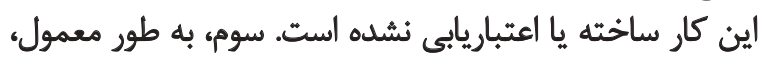

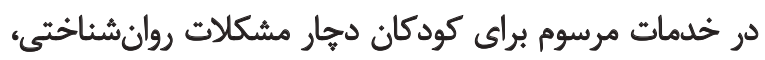

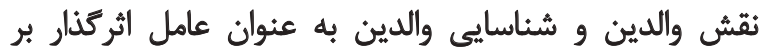

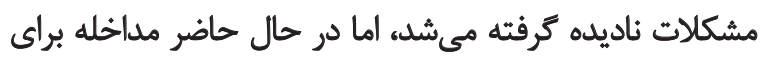

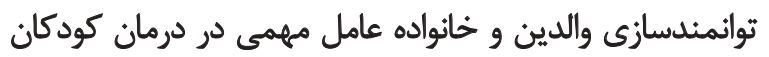

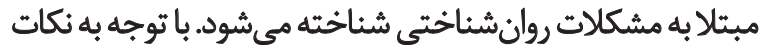

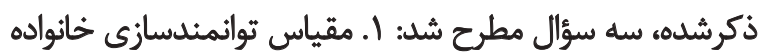

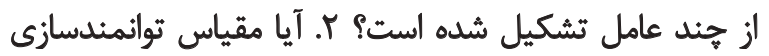

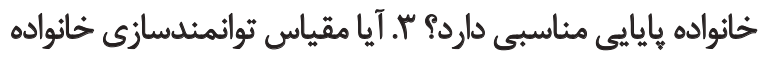

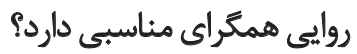
ووشُ روئ

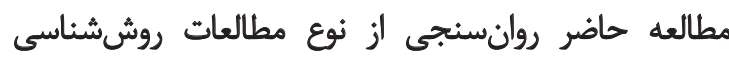

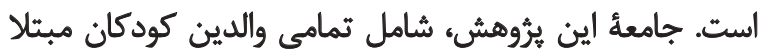

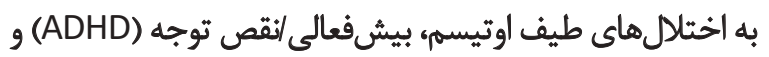

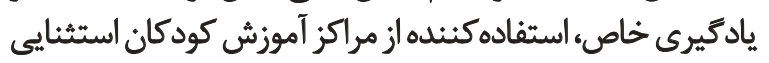

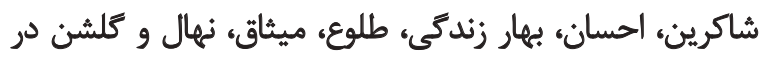

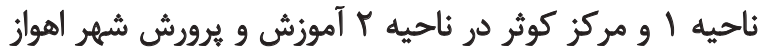

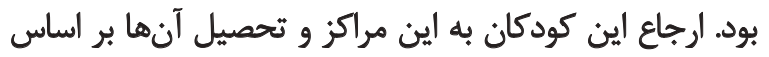

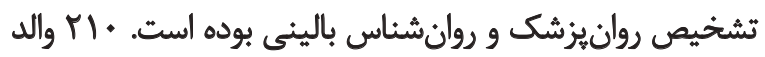

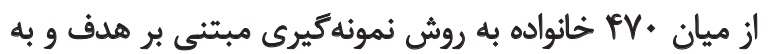

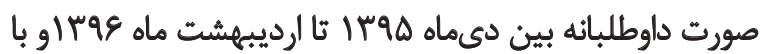

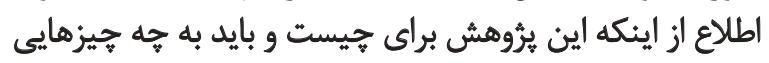
هاسخ بدهند، با رضايت كامل و شفاهى انتخاب شدين وائد. ملاك هاى ورود اين مطالعه عبارت بودند از: مدت ازدواج والدين

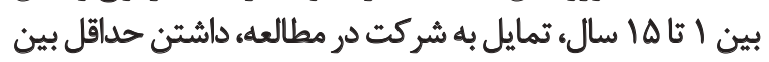

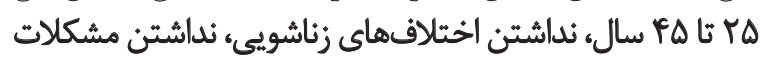

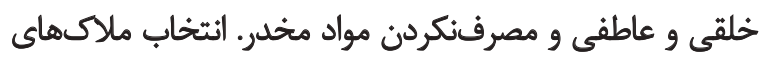

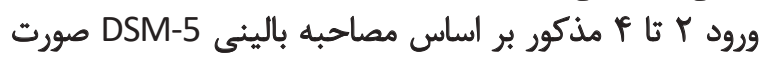

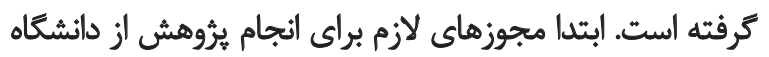

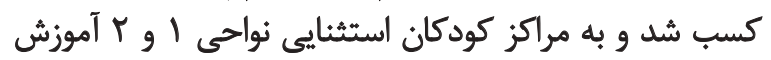

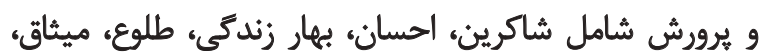

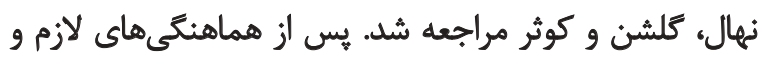

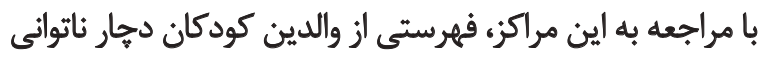

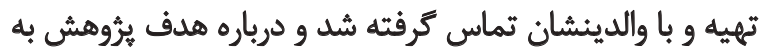

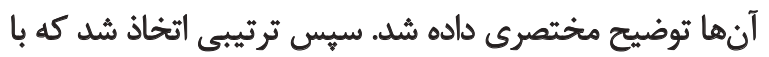

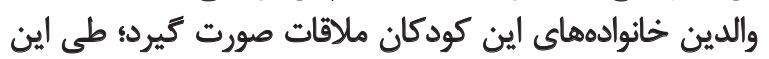




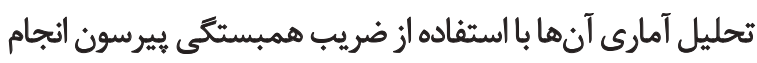

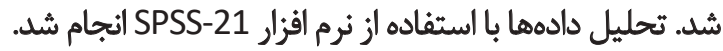

يافتهها

جدول شماره Y نشان مى بدهد ميانظين و انحراف معيار

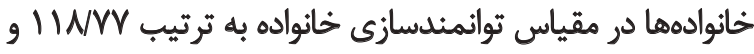
والم Tr/AT

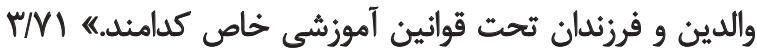

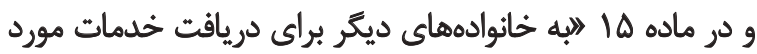

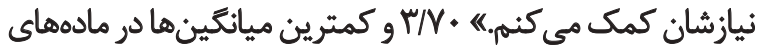

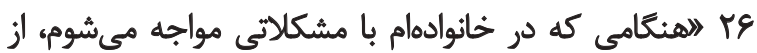

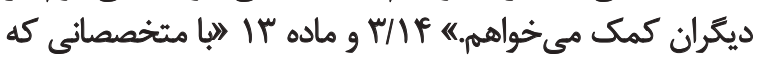

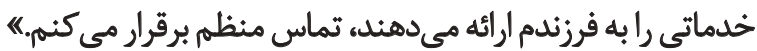

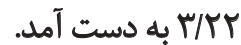

براى تحليل عاملى مقياس توانمندسازى خائواده در آغاز

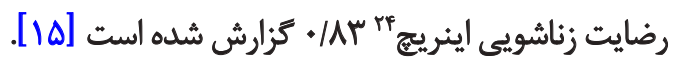

تحليل دادهها با استفاده از اين روشها انجام شد: ا. تحليل

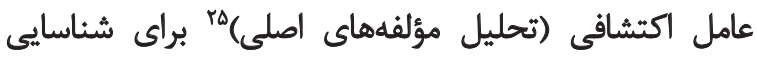

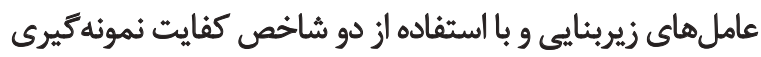

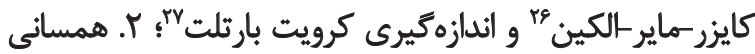

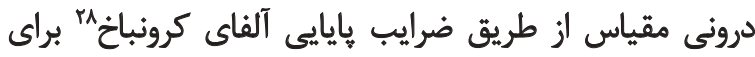

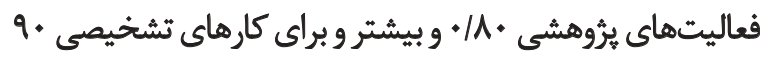

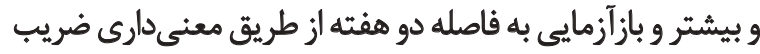

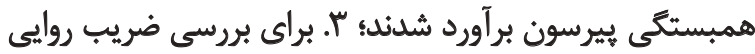

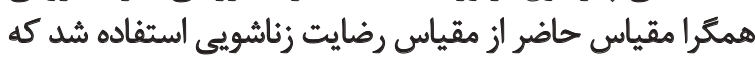

24. Enrich Marital Satisfaction Questionnaire

25. Principal components analysis

26. Kaiser-Meyer-Olkin

27. Bartlett's Test of Sphericity

28. Cronbach' $\alpha$ reliability

جدول ا. ويرُكى هاى جمعيتشناختى كروه نمونه

\begin{tabular}{|c|c|c|c|}
\hline فراوانى (درصد فراواتى) & حداقل (حداكثر) & مياتكَين (انحراف معيار) & مثغير \\
\hline- & $M(\varphi \varepsilon)$ & $\Gamma \Delta / \Gamma E(\Gamma / N \Delta)$ & سن \\
\hline- & $\Delta\left(M^{\prime}\right)$ & $1 . / T E(T / N \Delta)$ & مدت ازدواج \\
\hline $9 \Delta(f \Delta / T)$ & - & مرد & \\
\hline$\| \Delta(\Delta F / A)$ & - & ن & جنس \\
\hline$M \cdot(1+\infty)$ & - & 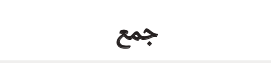 & \\
\hline$r \Delta(1 \& / V)$ & - & زير دييلم & \\
\hline$g q(r T / q)$ & - & دييلم & \\
\hline$P A(T \backslash / P)$ & - & فوقدييلم & \\
\hline A)(TE/M) & - & ليسأس & هيزان تحصيلات \\
\hline $9(\% / \pi)$ & - & فوق ليسانس & \\
\hline $1(\cdot / 0)$ & - & دكترى & \\
\hline$r)(1 .)$. & - & جمع & \\
\hline$V \Delta(r \Delta M)$ & & افرزند & \\
\hline$A V(P Y / P)$ & & ب بفرزند & \\
\hline $\operatorname{PA}(W N)$ & & س بفرزند & تعلداد فرزثلد \\
\hline $9(\% / m)$ & & أمرزند & \\
\hline $1(+/ \Delta)$ & & ه ف فرزئل و بيشتر & \\
\hline$m+(1 \cdot 0)$ & & 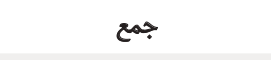 & \\
\hline$\Delta F(T \Delta / V)$ & & طيف اوتيسم & \\
\hline$r q(r V / g)$ & & بيش فعالى /نقص توجه & توع اختلال \\
\hline$W(r E M)$ & & يادييرى خاص & \\
\hline
\end{tabular}


جدول r. ميانكين و انتحراف معيار مادههاى مقياس توائمندسازى خانواده

\begin{tabular}{|c|c|c|c|c|c|c|c|c|c|}
\hline (انحرآفياتين معيار) & ماده内 & (انحراف معيانيثي) & ماده内ها & (انير افياتكين معيار) & مادهادها & (انجراف معياركين) & bostos & (أنحر ميافتئ معيار) & مادها L \\
\hline T/YN(/RA) & rq & $M / g r(I / M))$ & r & P/NA(Y/YA) & 10 & $r / M T\left(M / r_{0}\right)$ & $\wedge$ & $r / P f(1 / I)$ & 1 \\
\hline$r / E r(Y / / Q)$ & $r$ & $r / R F(W / T)$ & m & $r / F V(V / T \Lambda)$ & 18 & $r / \Delta T(Y / T \Delta)$ & 9 & r/AF(I/TA) & r \\
\hline$r / T r(Y / T E)$ & m & $H / M(M / N \cdot)$ & me & $M / \mathscr{R T}(\mathrm{V} / \mathrm{T})$ & IV & $r / \Delta \Delta(1 / T E)$ & 1. & $T / M N(Y /+F)$ & r \\
\hline$r / F+(1 / / F)$ & m & $r / R N(1 / / Q)$ & ro & $r / \Delta F(Y / / F)$ & IA & $r / m(I / I V)$ & 11 & $F / F I(Y / T H)$ & f \\
\hline$r / r e(1 / r q)$ & $m$ & m/NF(VE.) & re & $r / T \Delta(Y / \backslash A)$ & 19 & $M / T Y(1 / M)$ & ir & T/Yq(I/TY) & $\Delta$ \\
\hline T/AY(I/TY) & $m$ & $\operatorname{PRT}(1 / \circ)$ & TV & $m / 8 \cdot(1 / 19)$ & r. & $r / 8 \cdot(1 / \cdot r)$ & $\pi$ & $r / P V(Y / r)$ & 8 \\
\hline- & - & $H / f+(M / H)$ & ra & $r / f \Delta(V / \mu U)$ & ri & $r / V+(Y / T \cdot)$ & if & $r / F q(V / r q)$ & $\checkmark$ \\
\hline INWW(Tr/AT) & \multicolumn{9}{|c|}{ مجموع ميانكين (انحراف معيار) } \\
\hline
\end{tabular}

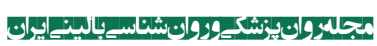

مادههاى مقياس توانمندسازى خانواده با حذف جهار ماده شامل

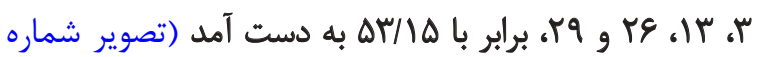

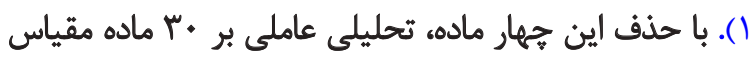

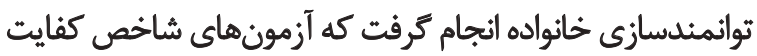

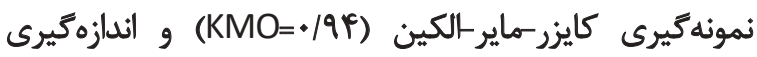

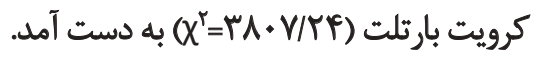

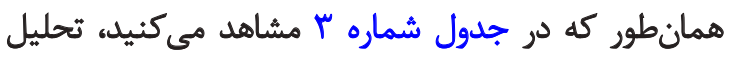

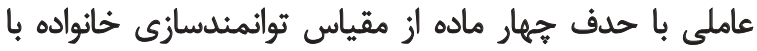

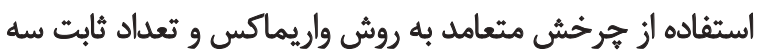

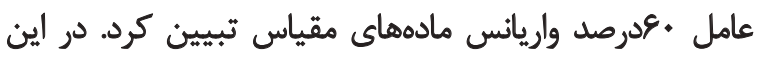

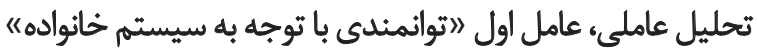

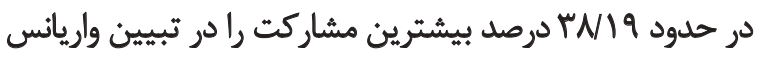

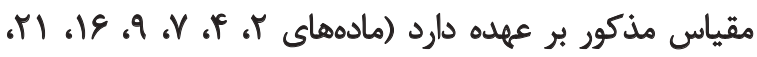

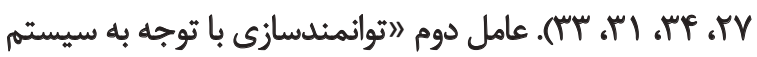

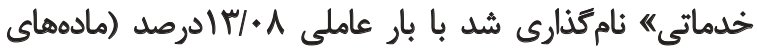

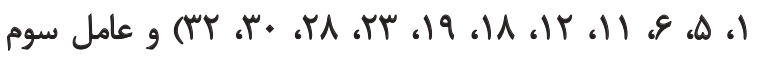

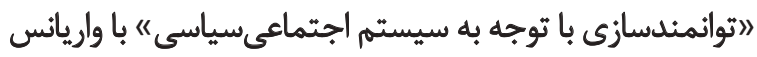

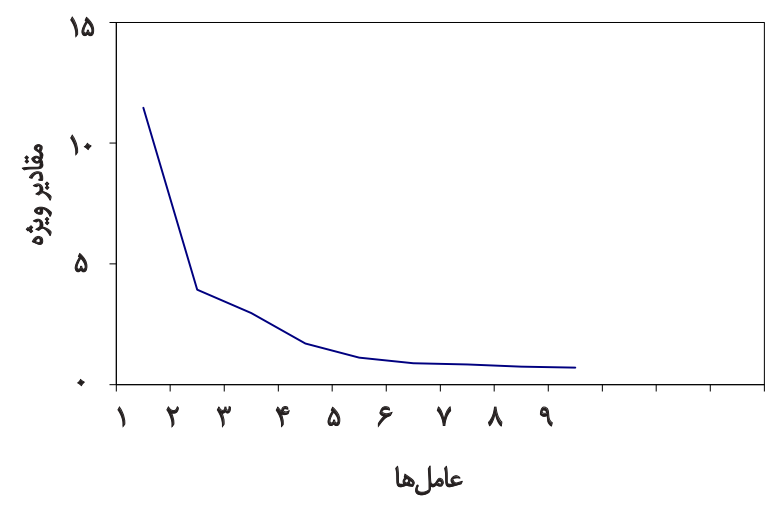

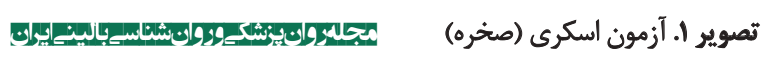

از روش تحليل مؤلفههاى اصلى و سيس براى تعيين عوامل

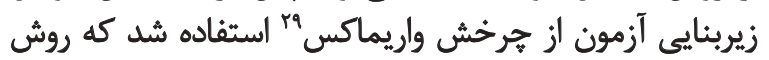

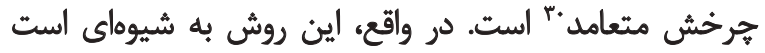

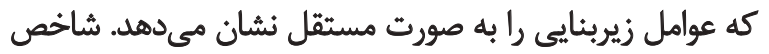

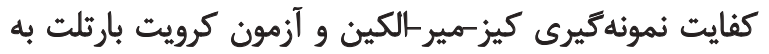

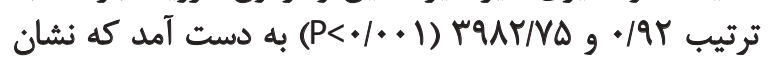

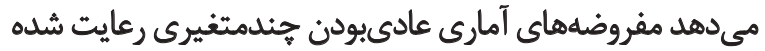

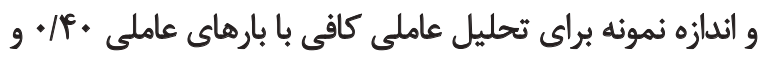

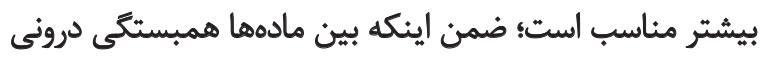
وجود ندارد و ماتريس همبستخى، ماتريس اتحاد است است.

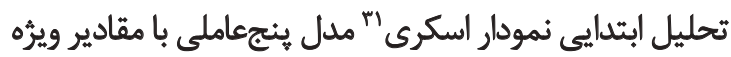

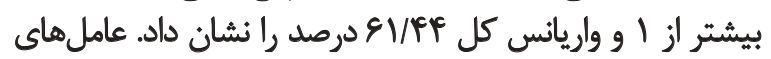

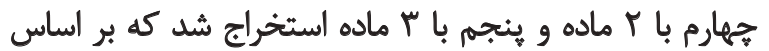

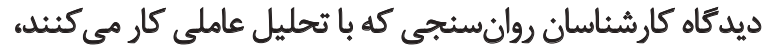

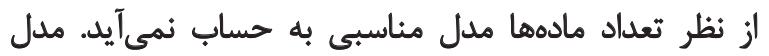

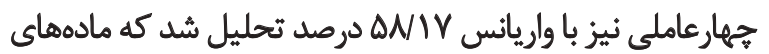

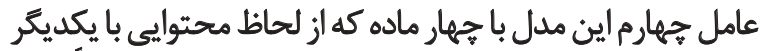

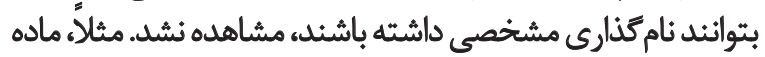

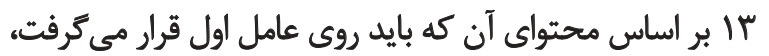
روى عامل جهارم قرار ترفت و به همين ترتيب سه ماده ديكر.

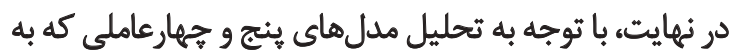

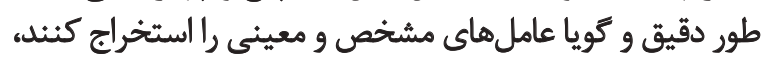

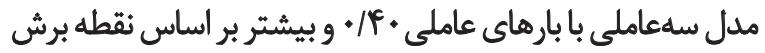

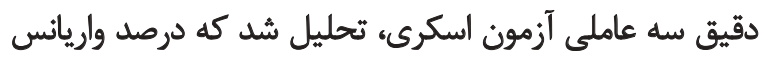

29. Varimax rotation

30. Orthogonal

31. Scree test 
جدول ஈ. ماتريس ساختار سهعاملى جرخشيافته واريماكس مرتبه دوم مقياس توانمثدسازى خانواده

\begin{tabular}{|c|c|c|c|c|c|c|c|c|c|c|c|}
\hline \multicolumn{3}{|c|}{ عامل } & \multicolumn{2}{|c|}{ بعد } & \multirow{2}{*}{ ماده } & \multicolumn{3}{|c|}{ عامل } & \multicolumn{2}{|c|}{ بعد } & \multirow{2}{*}{ ماده } \\
\hline سوم & دوم & اول & بيان & سطح & & سوم & دوم & اول & بيان & سطح & \\
\hline- & - &.$/ 9 P$ & نكرش & سيستم & $M$ & - & - & .19 & نكرش & سيستم & 1 \\
\hline- & - & $\cdot M$ & رفتار & سيستم & 19 & - & $\cdot / N A$ & - & رفتار & خاثوادكى & $r$ \\
\hline \multirow[t]{2}{*}{ T } & - & - & رفتار & اجتماعى & $r$. & \multicolumn{5}{|c|}{ حذف } & $r$ \\
\hline & 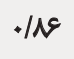 & - & نكرش & خائوادىى & $M$ & - &.$/ 8 V$ & & نكرش & خانواديى & $\varphi$ \\
\hline .198 & - & - & دانش & اجتماعى & r & - & - & .199 & دانش & سيستم & $\Delta$ \\
\hline - & - & $/ V$ & دانش & سيستم & $\pi$ & - & - &.$/ V$ & رفتار & سيستم & 8 \\
\hline . I\&A & - & - & دانش & اجتماعى & $r_{f}$ & - & NF & - & داشش & خانواد5ى & $r$ \\
\hline .181 & - & - & نكرش & اجتماعى & ro & d 194 & & - & رفتار & الجتماعى & 1 \\
\hline \multicolumn{5}{|c|}{ حذف } & $r q$ & - & $\cdot / W$ & - & نكرش & خانوادكى & 9 \\
\hline- & .190 & - & رفتار & خانوادّىى & iv & $\cdot M r$ & - & - & دانش & الجتماعى & 1. \\
\hline - & - &.$/ 94$ & رفتار & سيستم & $r A$ & $=$ & - & $\cdot M$ & داشش & الجتماعى & 11 \\
\hline \multicolumn{5}{|c|}{ حذف } & rq & - & - & .191 & دانش & سيستم & Ir \\
\hline- & & .189 & داشش & سيستم & $r$ & \multicolumn{5}{|c|}{ حذف } & r \\
\hline - & . $19 T$ & - & رقتتار & خائوادىى & $m$ & $\cdot M r$ & - & - & دانش & الجتماعى & if \\
\hline- & - & - & نكرش & سيستم & $\pi$ & $\cdot M r$ & - & - & رفتار & الجتماعى & 10 \\
\hline- & .181 & - & دانش & خانوادىى & $r$ & - & $\cdot M$ & & داش & خانوادكى & 18 \\
\hline- & .194 & - & نكرش & خانوادكى & $m$ & $\cdot M^{e}$ & - & - & نكرش & الجتماعى & iv \\
\hline
\end{tabular}

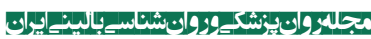

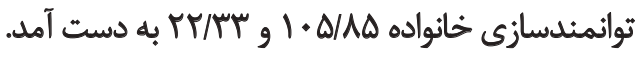

بحث

هدف اين يُؤهش، بررسى ويرُكى هاى روانسنجى نسخه

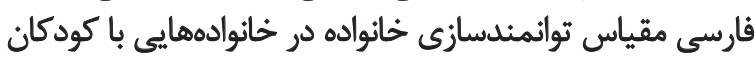

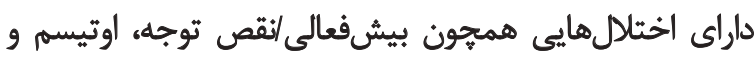

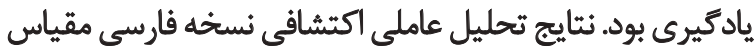

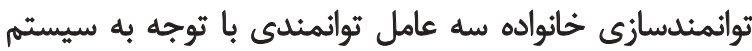

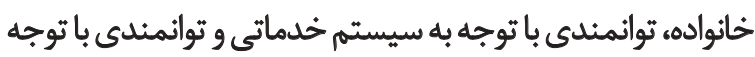

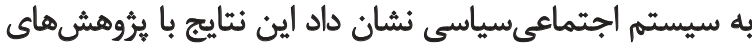

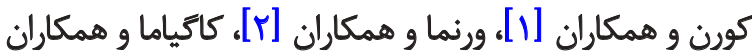

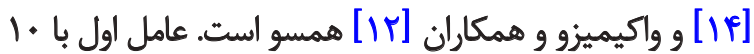

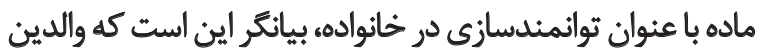

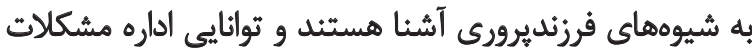

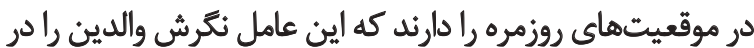

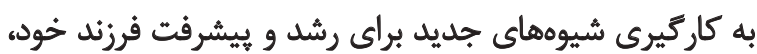

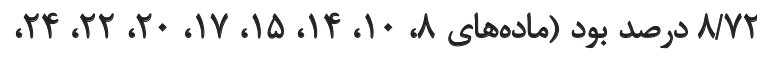

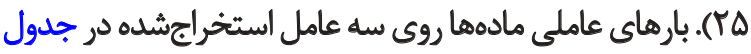

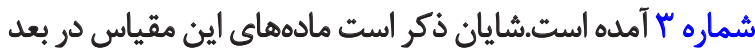

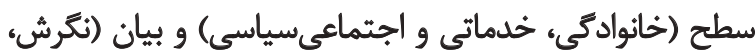

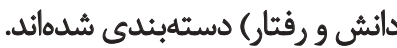

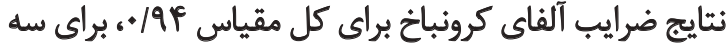

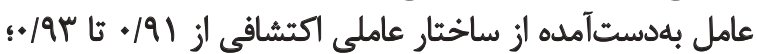

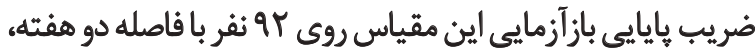

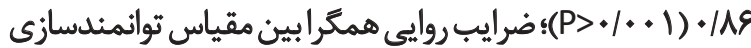

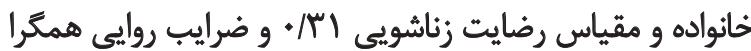

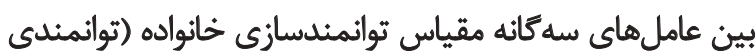

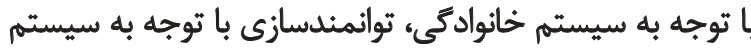

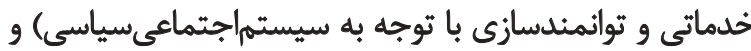

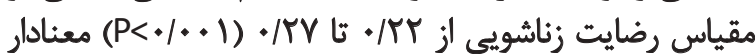

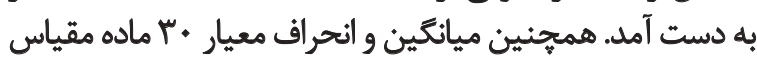


والدين در هر سه سطح توانمندسازى است. شواهد نشان مي دهد

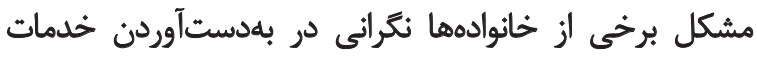

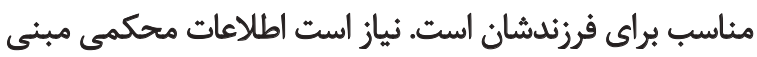

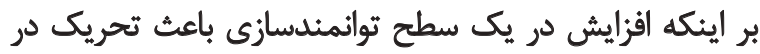

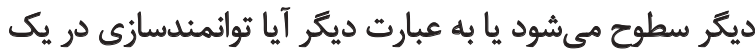

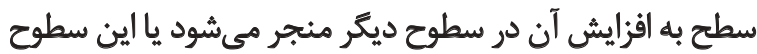
مجزا از هم هستند، كردآورى شود.

همكارى بيشتر با سيستم خدماتى با توانمندى زياد در ارتباط

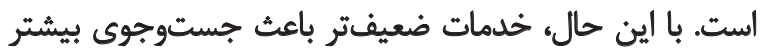

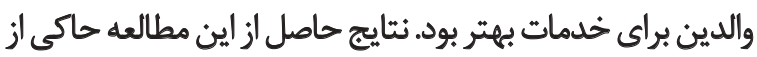

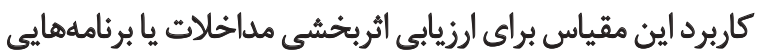

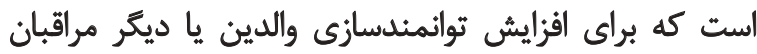

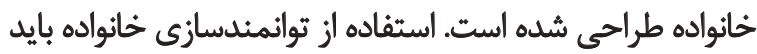

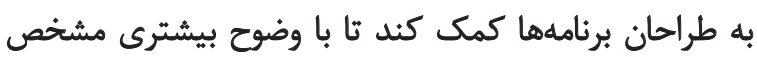

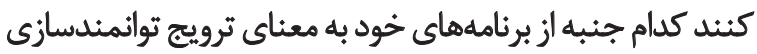

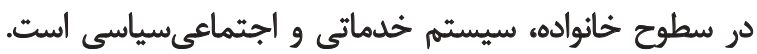

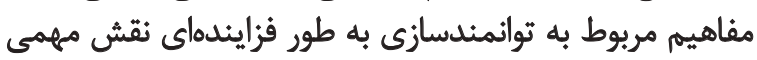

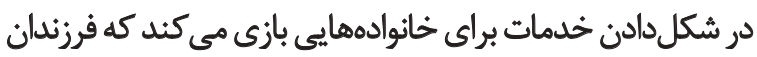

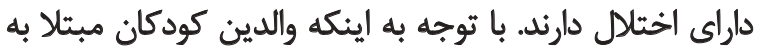

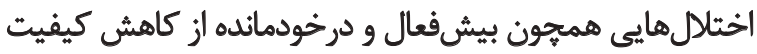

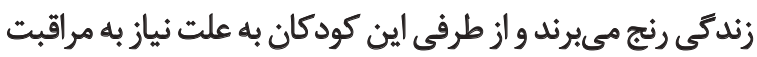

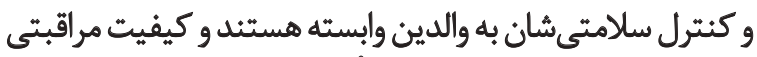

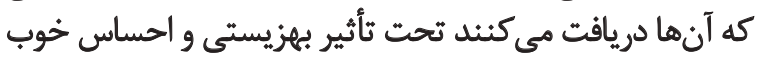

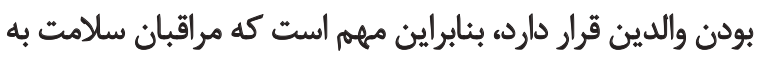

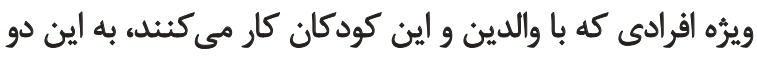
قشر توجه كافى داشته باشند.

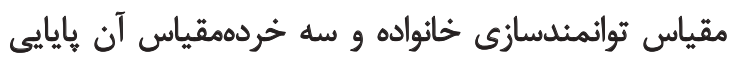

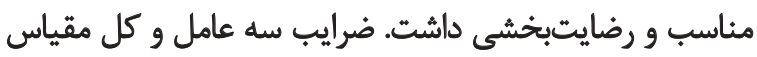

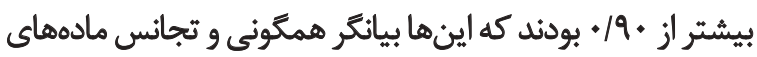

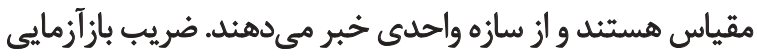

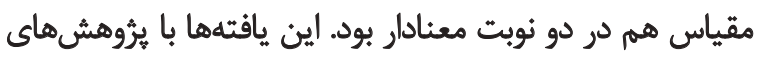

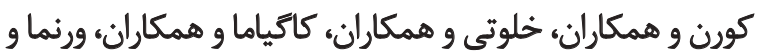

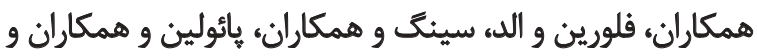

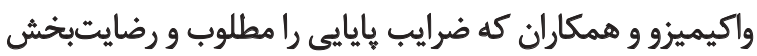

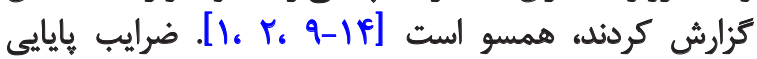

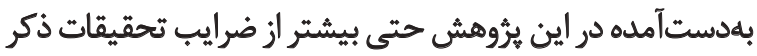

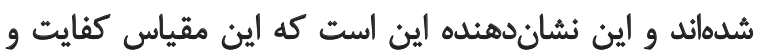

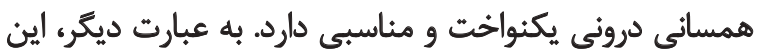

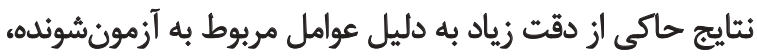

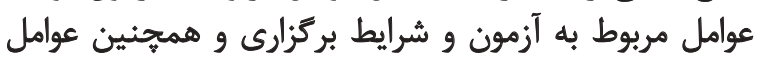

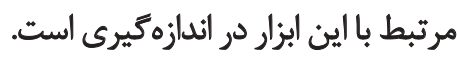

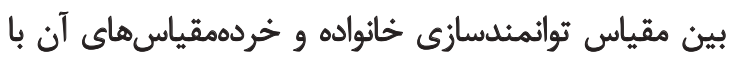

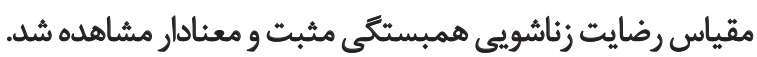

احساس خوب به والدبودن، دانش و آكاهي والدين از توانايىشان

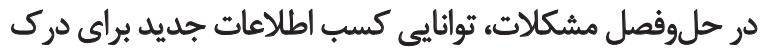

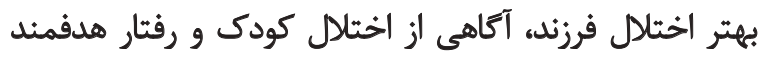

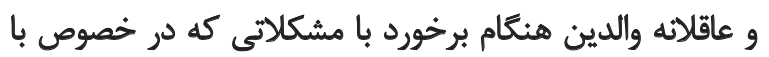
اختلال فرزندشان به وجود مى آيد، مى آرمايد.

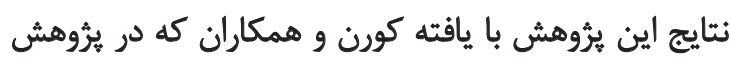

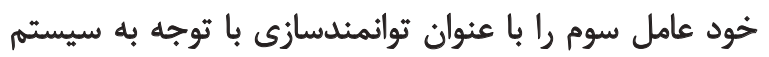

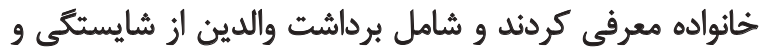

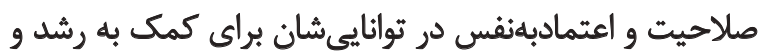

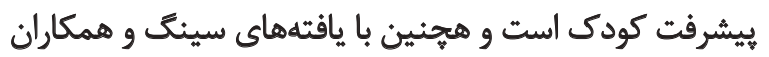

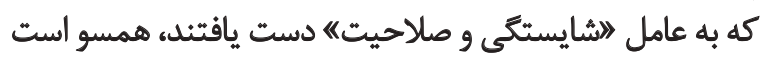

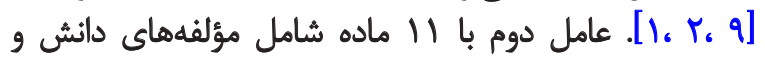

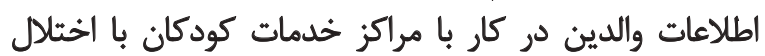

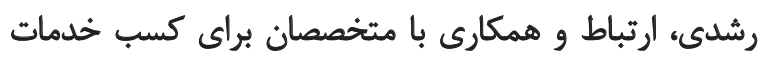

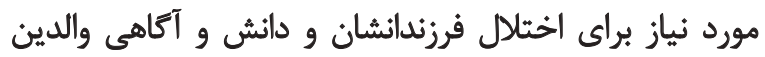

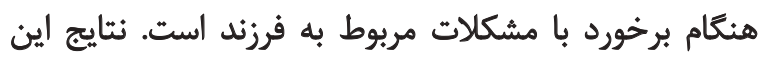

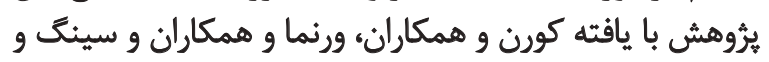

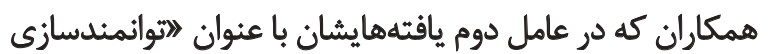

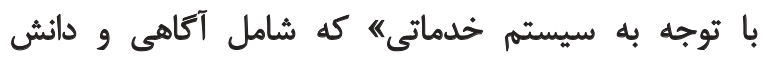

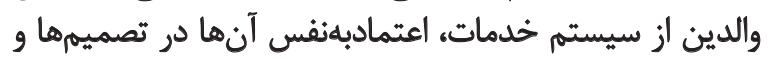

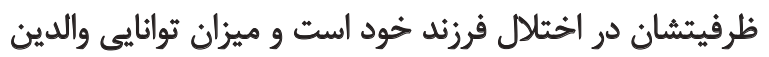

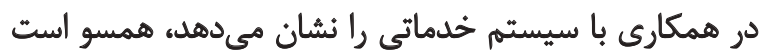
[I. Y. 9]

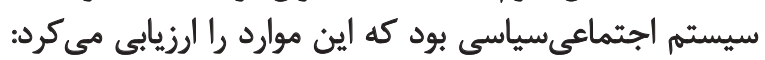

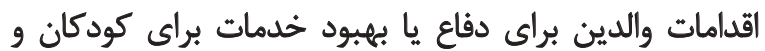

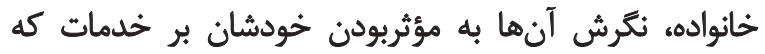

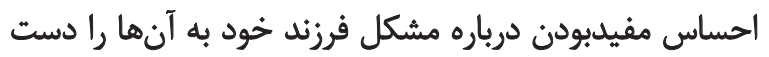

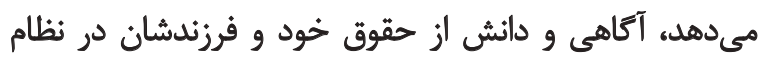

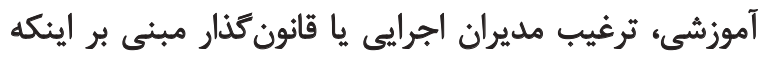

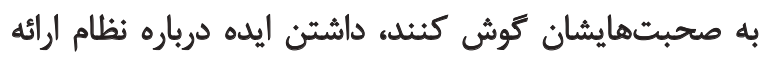

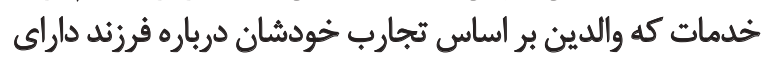

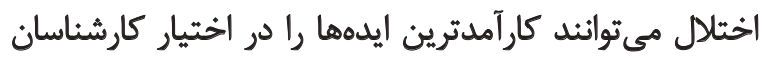

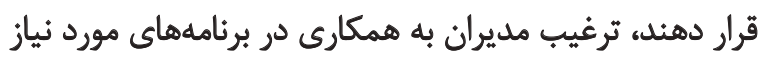

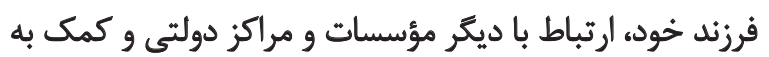

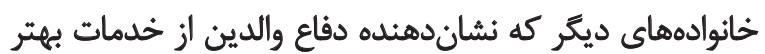

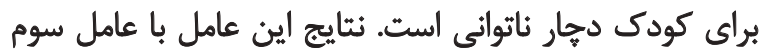

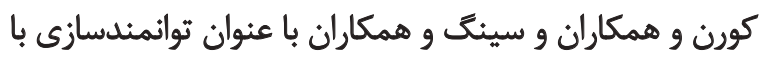

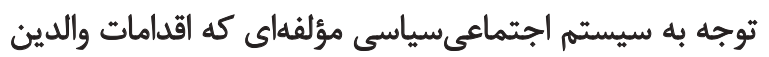

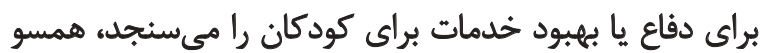

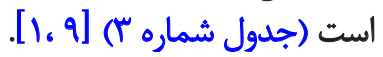

نمرههاى بيشتر در هر خردهمقياس منعكسكنينده سطوح

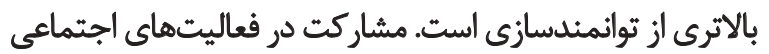

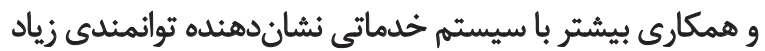




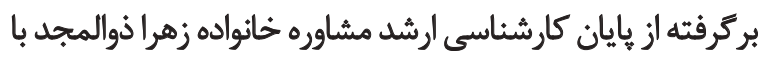
مجوز معاونت يروهشى دانشكاه شهيد جمران اندان اهواز است. بنا به اظهار نويسنده مسئول مقاله، حمايت مالى از يُروهش و تعارض منابع وجود نداشته است.
توانمندسازى خانواده به طور كلى به معناي نقطهة بوتها

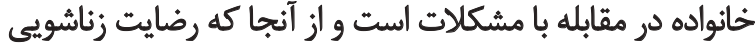

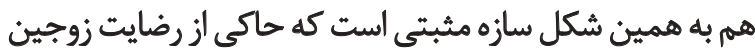

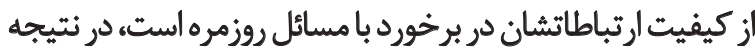

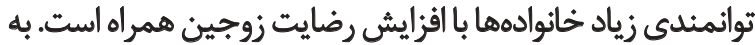

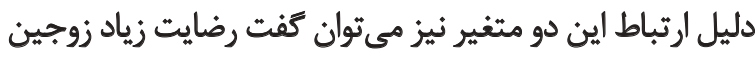

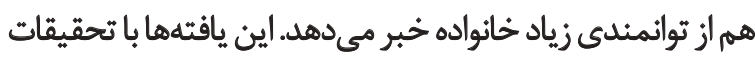

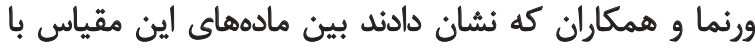

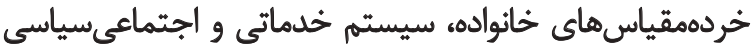

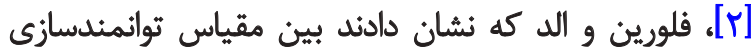

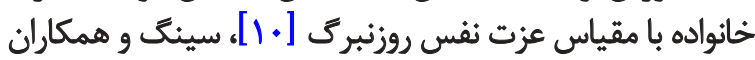

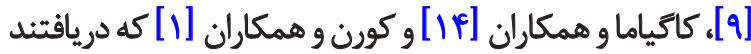

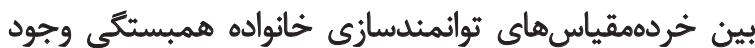

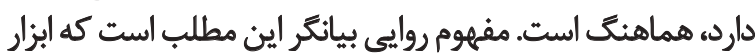
اندازهيرى تا جه حد خصيصه مد نظر را مى منسجد.

\section{نتيجلكيرى}

بنابراين بر اساس نتايج مىثوان از اين مقياس به عنوان ابزار

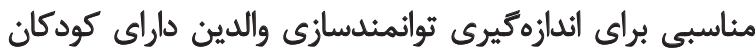

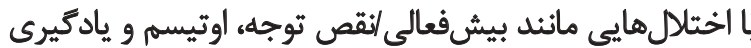

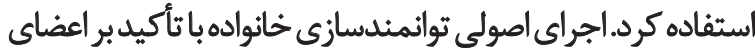

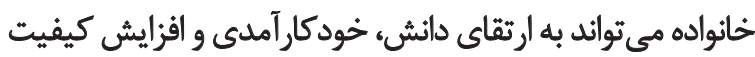

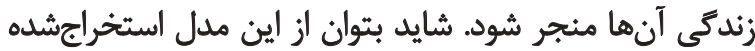

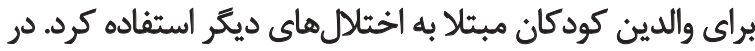

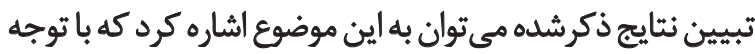

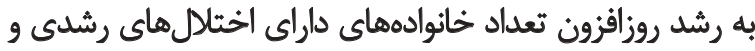

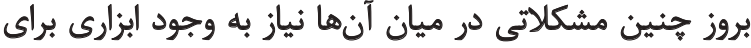

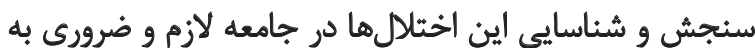

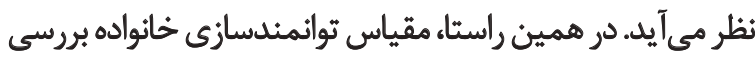

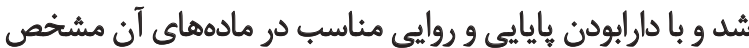

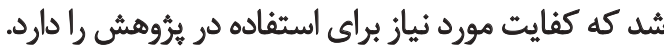
اين يُروهش ثنها به قشر خاصى از جامعه، يعنى والدين كودكان

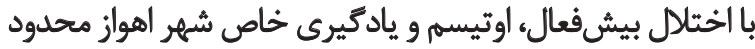

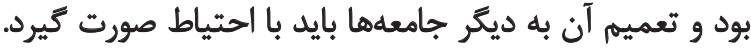

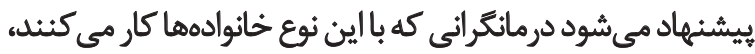

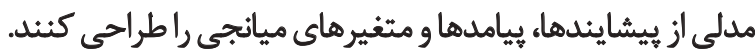

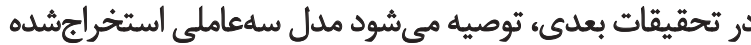
در جامعه ديكر بر اساس مدل تحليل توديل عاملى تأييدى بررسى شئ شود.

$$
\text { سياسكَّارى }
$$

بدين وسيله از همكارى مديريت مراكز آموزش كودكان

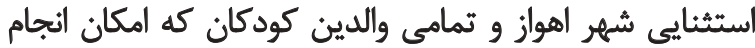
اين ثرؤهش را فراهم آوردند، صميمانه تشكر مى كنيمه اين مقاله 


\section{References}

[1] Koren PE, DeChillo N, Friesen BJ. Measuring empowerment in families whose children have emotional disabilities: A brief questionnaire. Rehabilitation Psychology. 1992; 37(4):305-21. [DOI:10.1037//0090-5550.37.4.305]

[2] Vuorenma M, Halme N, Astedt-Kurki P, Kaunonen M, Perala M. Validity and reliability of the personnel version of the Family Empowerment Scale (FES) for the social, health and educational services in Finland. Journal Advance Nurses. 2014; 70(4):927-36. [DOI:10.1111/jan.12242]

[3] Poertner J, Ronnau J. A strengths approach to children with emotional disabilities. In: Saleebey D, editor. The Strengths Perspective in Social Work Practice. New York: Longman; 1992. [PMID]

[4] Harrison T, Waite K, Hunter GL. The internet, information and empowerment, European Journal Marketing. 2006; 40(10):972-93. [DOI:10.1108/03090560610680961]

[5] Dunst CJ, Paget KD. Parent-professional partnerships and family empowerment. In: Fine MJ, editor. Collaboration With Parents of Exceptional Children. Brandon: Clinical Psychology Publishing; 1991.

[6] Sampsel D, McNichols S, Kordash RD, Bonitati D. Information for integration. A senior services program spurs development of a multi-hospital integrated network. Health Progress. 1994; 75(7):40-3. [PMID]

[7] Gutierrez L, Ortega R. Developing methods to empower Latinos: The importance of groups. Social Work Groups. 1991; 14(2):23-43. [DOI:10.1300/J009v14n02_03]

[8] Staples LH. Powerful ideas about empowerment. Administration Social Work. 1990; 14(2):29-42. [DOI:10.1300/J147v14n02_03]

[9] Singh NN, Curtis WJ, Ellis CR, Nicholson MW, Villani TM, Wechsler HA. Psychometric analysis of the Family Empowerment Scale. Journal of Emotional Behavioral Disorders. 1995; 3(2):85-91. [DOI:10.1177/106342669500300203]

[10] Florian V, Elad D. The impact of mothers' sense of empowerment on the metabolic control of their children with juvenile diabetes. Journal of Pediatric Psychology. 1998; 23(4):239-47. [DOI:10.1093/jpepsy/23.4.239]

[11] Pauline J, Jean M, Kruzich B, Friesen J, Robinson A. Family perceptions of participation in educational planning for children receiving mental health services. School of Social Work Journal. 2007; 32(1):75-92.

[12] Wakimizu R, Fujioka H, Yoneyama A, Iejima A, Miyamoto $\mathrm{S}$. Effectiveness of the group-based positive parenting program with Japanese families raising a child with developmental disabilities: A longitudinal study. Journal of Abnormal Child Psychology. 2014; 3:113. [DOI:10.4172/2329-9525.1000113]

[13] Khalvati M, Nafai A, Soltani M. [Relationship of family empowerment with metabolic control and adherence to treatment plans in children with phenylketonuria (Persian)]. Journal Kermanshah University of Medical Sciences. 2015; 18(8):491-97.

[14] Kageyama M, Nakamura Y, Kobayashi S, Yokoyama K. Validity and reliability of the Family Empowerment Scale for caregivers of adults with mental health issues. Journal of Psychiatric and Mental Health Nursing. 2010; 23(8):521-31. [DOI:10.1111/ jpm.12333]
[15] Rajabi Gh. [Factorial structural Marital Satisfaction Scale in marriage staff's members of Shahid Chamran University (Persian)]. Iranian Journal of Psychiatry \& Clinical Psychology. 2010; 15(4):359-67. 
مادهاي مقياس توانمندسازي خانواده
Losto

مادهاي مقياس تواثمندسازى خانواده

Lososto
خدماتى نيازز دارد، حائز الهميث است.

ديدكاه خود و آنهجه را كه درباره خلمات إرائهشده به فرزئدم تصور مي كنم، به كارشناسان مي مئميم.

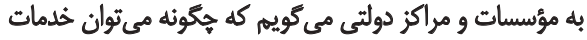

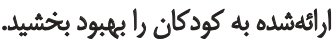

معتقدم مى توانم به همراه فرزندم، مشكلاتى را كه اثفاق مى اقتثلد.

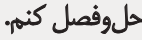

مي هاثم جكوئه هديران اجرائي يا قانون كذار را ترغيب كثم كه به

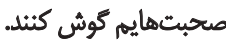

هيدانيم كه فرزندم هيه خلماتي نياز دارد.

مى دانم حقوق والدين و فرزئدان تحت قوائين أموزشى خاص كدامند.

احساس مى كنم دانش و تجربهام به عنوان يك والده مىثتوائد در

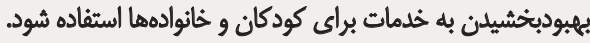

هنكامي كه در خانوادهام با مشكلاتي مواجه مي شومه از ديكران كمك مي خواهمبر مواته

سعى مى كنم شيوههاى جديد و نوينى را براى كمك به رشد فرزنلدم

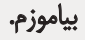

در صورت لزوم، در جستوجوى خدمات براى فرزئل و خانوادهام

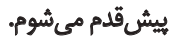

در برخورد با فرزندم به همان أندازه كه به مشكلات توجه دارم، به هيه جيزهاي خوب هم توجه هي كنم.

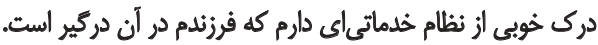

در هنكام برخورد با يك مشكل در رابطه با فرزنلدم، تصميم مي كيرم

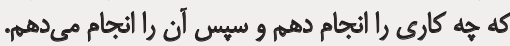

تصميم مى كيرم كه يه كارى را أنجام دهم و سيس آن را انجام

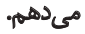

درك خوبي از اختلال كودكم دارم. - مري.

احساس مى كنم والد خوبى هستهم.

in

احساس مى كنم حق دارم تمامى خلماتى راكه فرزنلم دريافت مى كنله ثأييد كنم.

هنكامى كه مشكلاتي درباره فرزندم بروز مي كثند، بهخوبى آنها را

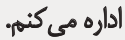

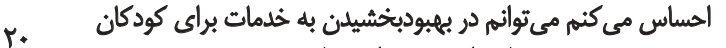

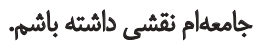

r)

بله توانايىام براى كمك به فرزندم كه رشد و ييشرفت كثلد، اعتماد

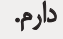

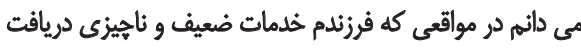

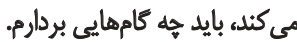

أز اين كه متخصصان نظراتم را درباره خدمات مورد نياز فرزئدم درى مي كنند، اطمينان خاطر يبيدا مي كنهم.

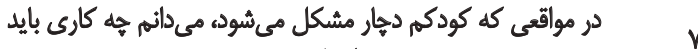

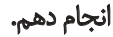

هنكامى كه لايحههاى مهمى درباره كودكان بله تصويب مىرسلد،

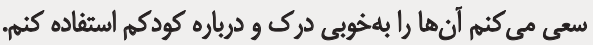

re

$$
\text { احساس مي كنم زندكى خانوادكىام تحت كثرل است. }
$$

rV

rq

r.

r

m

$m$

if
دركى مى كثم كه نظام ارائه خدمات به كودكان خمكوئه سازمان دهى

هي شود.

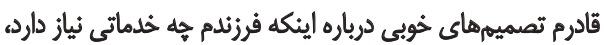

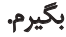

براى تصميميّي درباره خلمات مورد نياز فرزندم مى توانم با

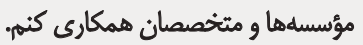

با متخصصانى كه خدماتى را به فرزندم ارائه مى دهنده تماس منظمه

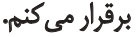

به خانوادههاي ديكر براى دريافت خدمات مورد نيازشان كمى

مى كنم

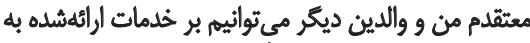

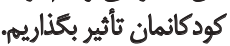

ir

if 
